\title{
Direction of arrival estimation by cross-power spectrum phase analysis using prior distributions and voice activity detection information
}

\author{
Yuuki Tachioka*, Tomohiro Narita ${ }^{\dagger}$ and Tomohiro Iwasaki ${ }^{\ddagger}$ \\ Information Technology $R \&$ D Center, Mitsubishi Electric Corporation, 5-1-1 Ofuna, Kamakura, 247-8501 Japan
}

(Received 8 August 2011, Accepted for publication 6 September 2011 )

Keywords: Sound source localization, DOA estimation, CSP analysis, Noise robustness, Prior distributions PACS number: 43.60.Fg, 43.60.Jn [doi:10.1250/ast.33.68]

\section{Introduction}

Sound source localization is important as a pre-process for target detection, speech enhancement, and speech recognition. To estimate the direction of arrival (DOA), the cross-power spectrum phase (CSP) analysis that uses two microphones is widely known as an effective estimation method [1]. However, the accuracy of the CSP analysis decreases at low SNR and owing to directional noise. Denda proposed a weighted CSP analysis, that weighs the spectrum in speech bands, and CSP coefficient subtraction that subtracts estimated noise components from CSP coefficients [2]. These methods are applied only for speech with stationary noise. Nishiura proposed synchronous addition of a CSP coefficient, which uses three or more microphones and synchronously adds paired CSP coefficients [3]. This increases device size and computational load. In this paper, we propose a CSP analysis using prior distributions of source direction and voice activity detection (VAD) information in order to eliminate noise from CSP coefficients. This method can be adopted for any sound sources if activity of the source can be detected somehow, and requires minimal computation because the algorithm is simple and realized by two microphones.

\section{Eliminating noises from CSP coefficients}

2.1. Outline of CSP analysis

In the CSP analysis, DOA is estimated from the arrival time delay $\tau[\mathrm{s}]$ using a cross-power spectrum between the two microphones. The distance between the microphones is $L_{\mathrm{m}}$ [m]. First, CSP coefficient $\operatorname{CSP}(i, k)$ is obtained as in Eq. (1):

$$
\operatorname{CSP}(i, k)=\mathcal{F}_{i}^{-1}\left(\frac{\mathcal{F}_{i}\left(x_{1}(t)\right) \mathcal{F}_{i}\left(x_{2}(t)\right)^{*}}{\left|\mathcal{F}_{i}\left(x_{1}(t)\right)\right|\left|\mathcal{F}_{i}\left(x_{2}(t)\right)\right|}\right),
$$

where $\mathcal{F}_{i}$ is short-time Fourier transform (STFT) at frame number $i$, and $*$ denotes a complex conjugate. $x_{1}$ and $x_{2}$ denote the inputs of two microphones. CSP coefficients are a function of the delay time $k\left(0 \leq k \leq k_{\max }=\operatorname{INT}\left(L_{\mathrm{m}} f_{\mathrm{s}} / c\right)+1\right)$. $f_{\mathrm{s}}$ denotes a sampling frequency $[\mathrm{Hz}], c$ denotes a sound speed $[\mathrm{m} / \mathrm{s}]$, and INT is a function that returns the integer parts of arguments.

Second, the arrival time delay $\tau$ is represented as a peak of CSP coefficient and is calculated as follows: $\tau=$

\footnotetext{
*e-mail: Tachioka.Yuki@eb.MitsubishiElectric.co.jp

†e-mail: Narita.Tomohiro@ct.MitsubishiElectric.co.jp

‡e-mail: Iwasaki.Tomohiro@bp.MitsubishiElectric.co.jp
}

$\arg \max _{k}(C S P(i, k))$ [1]. Finally, the direction of the sound source $\theta$ is obtained as $\theta=\sin ^{-1}\left(\tau c /\left(L_{\mathrm{m}} f_{\mathrm{s}}\right)\right)$.

Figure 1 shows a schematic diagram of the proposed method. In this paper, we define "CSP(I)" and "CSP(II)" as baseline methods. "CSP(I)" is an original CSP analysis. "CSP(II)" is a conventional CSP analysis with a peak-hold process [4] and noise component suppression, which sets the cross power spectrum to zero when the estimated SNR is under $0 \mathrm{~dB}$. The proposed method, which we name "CSP F," has a CSP coefficient filtering process that is added to "CSP(I)" and "CSP(II)" respectively in Sects. 2.2 and 2.3.

\subsection{Using prior distributions}

We assume that sources do not move substantially and the duration of sources is longer than that of noise. For example, when you operate hands-free devices by voice, the location of speaker would not move substantially. Smoothed CSP coefficient $\overline{C S P}(i, k)$ is obtained as in Eq. (2) by averaging $\operatorname{CSP}(i, k)$ during $2 d+1$ frames (here, $d=5$ ).

$$
\overline{C S P}(i, k)=\frac{1}{2 d+1} \sum_{j=i-d}^{i+d} \operatorname{CSP}(j, k) .
$$

We assume that $\overline{C S P}(i, k)$ is a likelihood of sound source directions, which correspond to a delay time $k$, then we obtain an accumulated likelihood $L(i, k)$ as follows: $L(i, k)=$ $\sum_{j=0}^{i+d} \operatorname{CSP}(j, k)$. Prior distribution $P(i, k)(0 \leq P(i, k) \leq 1)$ is normalized by the maximum value of $L(i, k)$, as in Eq. (3):

$$
P(i, k)=\frac{\operatorname{MAX}(L(i, k), 0)}{\operatorname{MAX}\left(L(i, 0), L(i, 1), \cdots, L\left(i, k_{\max }\right)\right)},
$$

where MAX is a function that returns a maximum value of arguments. Finally, the filtered CSP coefficient $\overline{\operatorname{CSP}}^{\prime}(i, k)$ is obtained by combining a weighted CSP coefficient whose weight is $P(i, k)$ with an original coefficient at the combination ratio $r$, as in Eq. (4):

$$
\overline{C S P}^{\prime}(i, k)=(r+(1-r) P(i, k)) \overline{C S P}(i, k) .
$$

If source does not move and SNR is high, simple averaging as $\overline{C S P}^{\prime \prime}(i, k)=\sum_{j=0}^{i+d} \operatorname{CSP}(j, k) /(i+d+1)$ is effective. However, if large CSP value of noise inputs, estimation accuracy decreases significantly because the peak of noise hardly diminishes by averaging. The proposed method only suppresses the noise component and does not increase the peak value of CSP coefficient because $P(i, k)$ is 1 or less. Hence, the 


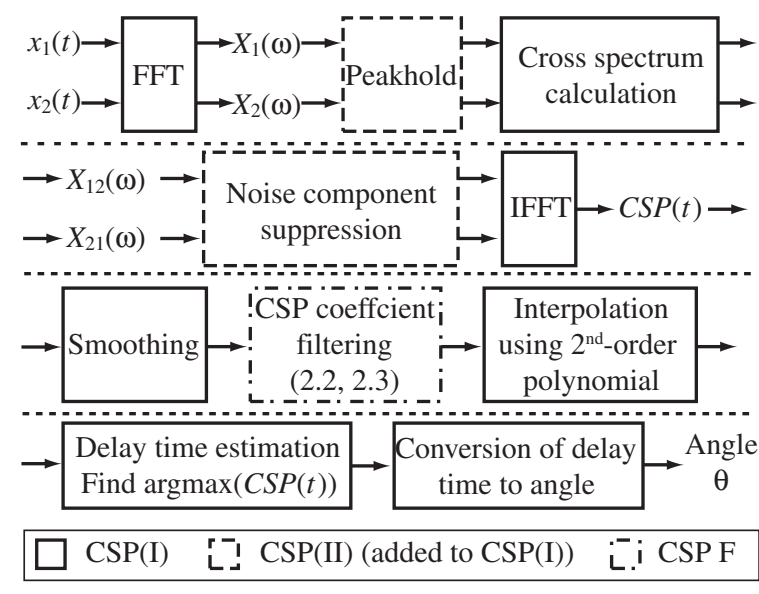

Fig. 1 Schematic diagram of the proposed method.

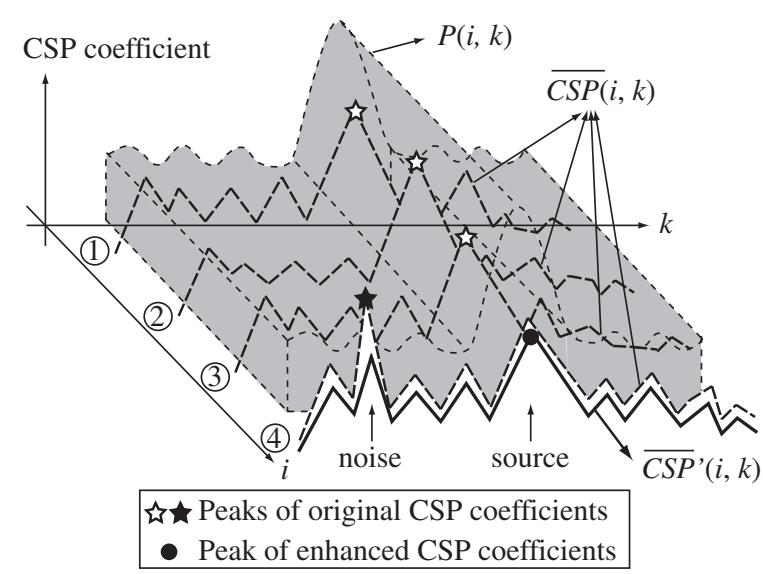

Fig. 2 Procedure for calculating a likelihood function of CSP coefficients and eliminating noise.

proposed method is less affected by noise than the simple averaging.

Figure 2 clearly shows the above-mentioned procedure. We obtain $\overline{C S P}(i, k)$. The source direction is the center, but a noise peak appears on the left side in the 4 th-frame. This peak is indicated as a closed asterisk in Fig. 2. If the source does not move substantially, the center peak is reliable because the difference between the noise peak and the center peak is small. Then, we calculate $P(i, k)$ using accumulated CSP coefficients, as in Eq. (3). The central section of a likelihood function is greater than other sections, because previous peaks have appeared at the center. We localize the source at the center, indicated by a closed circle, by multiplying $P(i, k)$ and $\overline{C S P}(i, k)$ and combining these and the original CSP coefficients at the ratio $r$, as in Eq. (4).

2.3. Using voice activity detection information

If the target is speech, peaks of CSP coefficients in nonspeech areas are attributed to noise. A modified likelihood $L^{\prime}(i, k)$ is obtained as in Eq. (5) according to VAD information.

$$
L^{\prime}(i, k)=\sum_{j=0}^{i+d}((1+\alpha) \delta(j)-\alpha) \operatorname{CSP}(j, k),
$$

where $\delta(j)$ is a function that returns unity in speech areas and zero in non-speech areas at frame $j$, and $\alpha(\alpha>0)$ is a penalty.

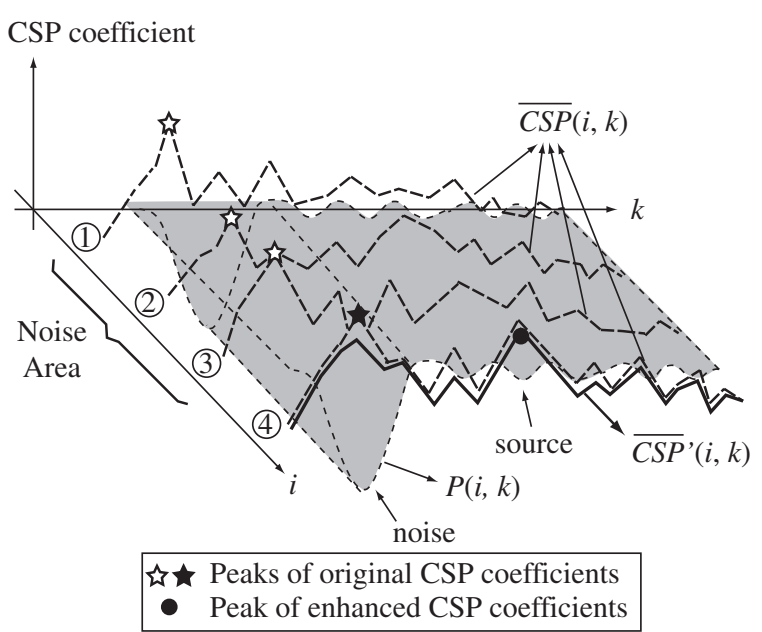

Fig. 3 Procedure for calculating a likelihood function of CSP coefficients with VAD information and eliminating the disturbance of noise.

This leads to a sign inversion of CSP coefficients in non-speech areas. Peaks of speech are enhanced by suppressing noise peaks, which are dominant in non-speech areas. Figure 3 illustrates this procedure. The first three frames are noise according to VAD. The speech peak appears at the center in the 4th-frame. However, the noise peak on the left side indicated as a closed asterisk is higher. In this case, likelihood $L^{\prime}(i, k)$ is obtained as in Eq. (5) by inverting any sign of CSP coefficients in the noise area and $P(i, k)$ is estimated from these likelihoods. The speech peak is enhanced and a source is located as a closed circle using the same procedure as in Eq. (4).

\section{Experiments}

\subsection{Experimental setup}

Impulse responses were measured at every $30^{\circ}$ at the center and near the wall of the room as shown in Fig. 4. The minimum and maximum distances between sources and the center of a microphone array ( $8 \mathrm{ch}$ circle array) $L_{s r}$ were 1 and $2 \mathrm{~m}$, respectively. Reverberation time $T_{30}$ was $0.68 \mathrm{~s}$ and the reverberation decay curve was not bent. Evaluation data was produced by convolving speech (control words for air conditioner) with these impulse responses. Speaker direction was located using two diagonal microphones. Recorded noise (Table 1) was added to the evaluation data at SNR of 6 and $24 \mathrm{~dB}$. Sampling frequency $f_{\mathrm{s}}$ was $16 \mathrm{kHz}$, and the window length and frame shift of STFT were $60 \mathrm{~ms}$ and $30 \mathrm{~ms}$, respectively. According to preparatory studies, $r$ was 0.3 [Eq. (4)] and $\alpha$ was 1.0 [Eq. (5)]. Note that performance depended little on these parameters.

3.2. Results and discussions

3.2.1. Comparison with the two-microphone method

Estimation accuracy in the speech area is calculated by each frame when the microphone array is located at the center of the room. The error tolerance is $\pm 15^{\circ}$. The estimation average accuracy in all directions is shown in Figs. 5 and 6, which represent the easiest case (SNR of $24 \mathrm{~dB}, L_{s r}$ of $1 \mathrm{~m}$ ) and the most difficult case (SNR of $6 \mathrm{~dB}, L_{s r}$ of $2 \mathrm{~m}$ ), respectively. A combination of " $\mathrm{CSP}(\mathrm{I})$ " and the proposed method ("CSP F") improves the accuracy in Fig. 5 but not in 


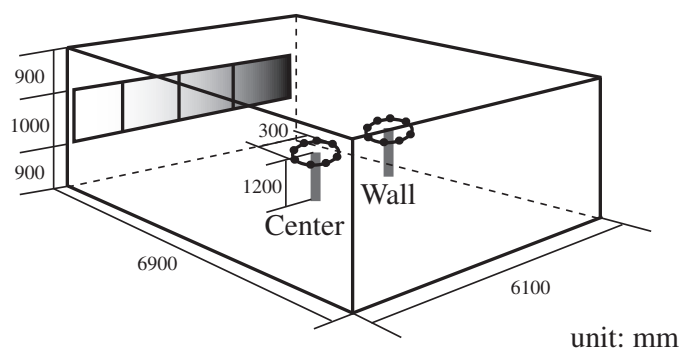

Fig. 4 Geometry of a meeting room.

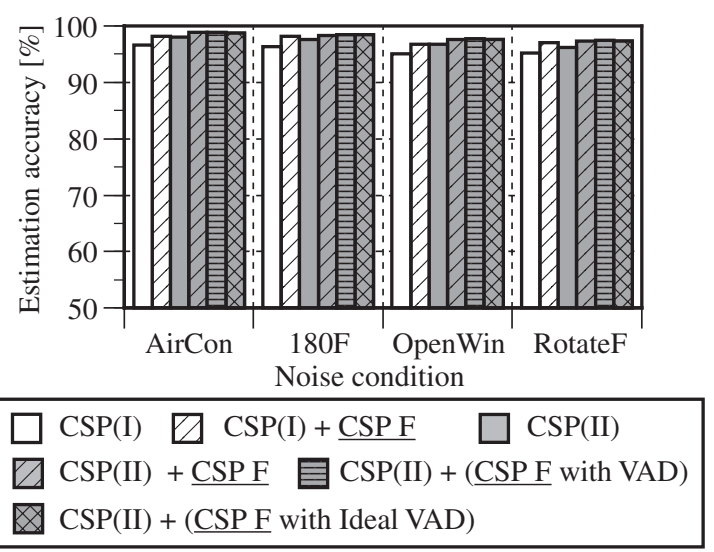

Fig. 5 Estimation accuracy of arrival direction when SNR is $24 \mathrm{~dB}$ and the distance between the source and the center of the microphone array $L_{s r}$ is $1 \mathrm{~m}$.
Table 1 Recorded noise that is added to evaluation data. (SNR of 6 and $24 \mathrm{~dB}$ )

\begin{tabular}{ll}
\hline \multicolumn{1}{c}{ Name } & \multicolumn{1}{c}{ Explanation } \\
\hline AirCon & Air Conditioner noise. \\
$180 \mathrm{~F}(270 \mathrm{~F})$ & Foot noise generated at $180^{\circ}\left(270^{\circ}\right)$. \\
OpenWin & Environmental noise with windows open. \\
RotateF & Foot noises rotating \\
& around the microphone array. \\
\hline
\end{tabular}

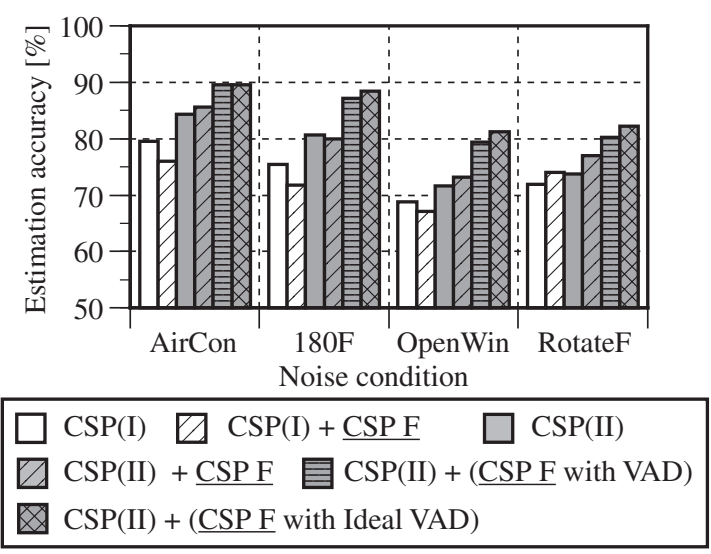

Fig. 6 Estimation accuracy (SNR of $6 \mathrm{~dB}, L_{s r}$ of $2 \mathrm{~m}$ ).
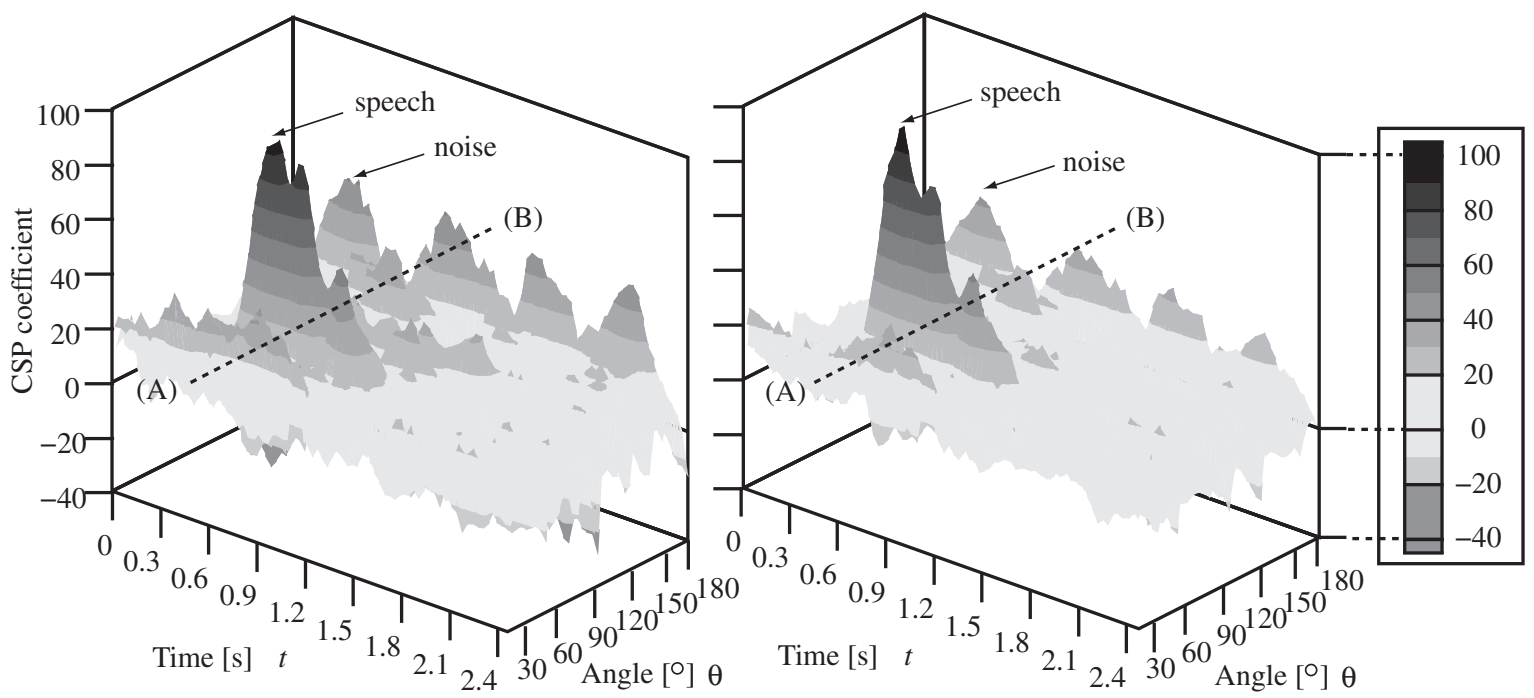

Fig. 7 Original and enhanced CSP coefficients on the time-angle plane. (left: original, right: enhanced)

Fig. 6, because of less accurate prior distributions when SNR is low. Accuracy increases with a combination of "CSP(II)" and "CSP F," because the noise components of the cross power spectrum are reduced in advance. In addition, the use of VAD [5] improves the performance of "CSP F" because the estimation accuracy of prior distributions increases as noises are learned ("CSP F with VAD"). The difference in accuracy between automatic VAD and manually tagged VAD ("CSP F with Ideal VAD") is not large.
Figure 7 shows CSP coefficients on a time-angle plane with directional noise at "180F" and speech at $60^{\circ}$ (SNR of $6 \mathrm{~dB}, L_{s r}$ of $2 \mathrm{~m}$ ). Using the proposed method, maximal peaks created by foot noises near $180^{\circ}$ are suppressed, maintaining the speaker peaks near $60^{\circ}$, as seen on the right graph, unlike that observed for the peaks of the conventional method, as seen on the left graph. Figure 8 shows the section at A-B (1.02 s) of Fig. 7. Originally, the peak appears close to $160^{\circ}$, but with the proposed method, it is correctly estimated closed to $60^{\circ}$. 


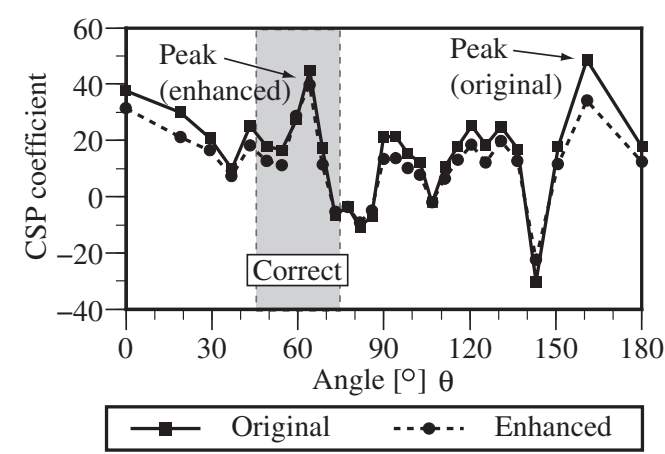

Fig. 8 Enhanced CSP coefficient $(t=1.02[\mathrm{~s}])$.

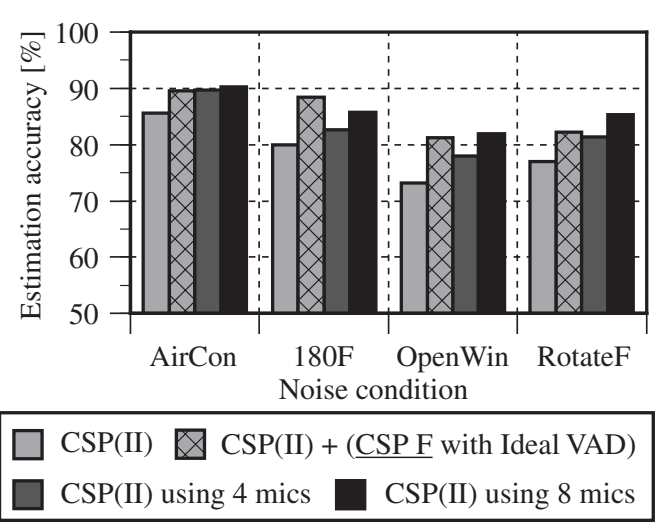

Fig. 9 Estimation accuracy of the proposed method (SNR of $6 \mathrm{~dB}, L_{s r}$ of $2 \mathrm{~m}$ ) compared with that using four and eight microphones.

3.2.2. Comparison with four- and eight-microphone methods

Using three or more microphones reduces noise by synchronously adding paired CSP coefficients [3]. Figure 9 shows the results using four microphones (all six pairs) and eight microphones (four diagonal pairs). The accuracy of the proposed method is superior to the method that uses four microphones and is equivalent to the method that uses eight microphones.

3.2.3. Effect of receiving point

Figure 10 shows the accuracy when the microphone array is near the wall (SNR of $6 \mathrm{~dB}, L_{s r}$ of $2 \mathrm{~m}$ ). Although it is difficult to estimate DOA because 1st-order reflected sounds

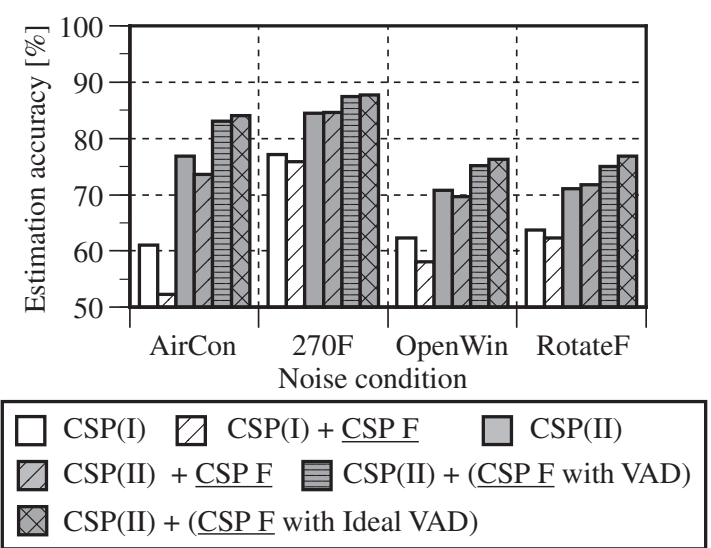

Fig. 10 Estimation accuracy (SNR of $6 \mathrm{~dB}, L_{s r}$ of $2 \mathrm{~m}$ ). Sources and receivers are located near the wall.

are stronger, estimation accuracy is improved by using a combination of "CSP(II)" and "CSP F with VAD."

\section{Conclusions}

We propose a method that reduces the effect of noise for the estimation of DOA by CSP analysis. The proposed method uses prior distributions estimated from accumulated CSP coefficients. We demonstrated that this method was effective for both diffusive and directional noise and that using VAD information improved estimation accuracy.

\section{References}

[1] C. Knapp and G. Carter, "The generalized correlation method for estimation of time delay," IEEE Trans. Acoust. Speech Signal Process., 24, 320-327 (1976).

[2] Y. Denda, T. Nishiura and Y. Yamashita, "Robust talker direction estimation based on weighted CSP analysis and maximum likelihood estimation," IEICE Trans., E89-D, 10501057 (2006).

[3] T. Nishiura, T. Yamada, T. Nakamura and K. Shikano, "Localization of multiple sound sources based on a CSP analysis with a microphone array," IEEE Int. Conf. Acoust. Speech Signal Process., Vol. 2, pp. 1053-1056 (2000).

[4] T. Suzuki and Y. Kaneda, "Sound source direction estimation based on subband peak-hold processing," J. Acoust. Soc. Jpn., 65, 513-522 (2009).

[5] J. Sohn, N. S. Kim and W. Sung, "A statistical model-based voice activity detection," IEEE Signal Process. Lett., 6, 1-3 (1999). 